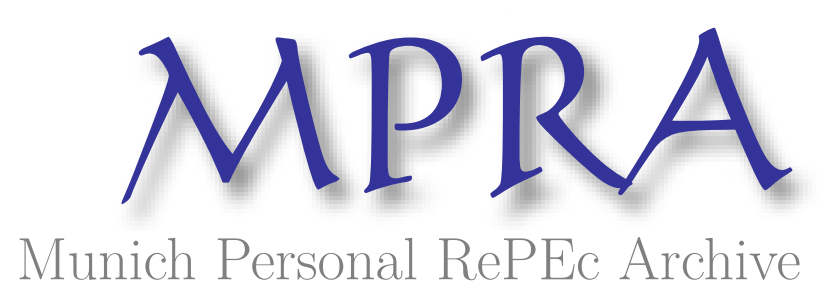

\title{
Evolutionary Model of Non-Durable Markets
}

Kaldasch, Joachim

EBC Hochschule Berlin

27 September 2011

Online at https://mpra.ub.uni-muenchen.de/33743/

MPRA Paper No. 33743, posted 27 Sep 2011 12:41 UTC 


\title{
Evolutionary Model of Non-Durable Markets
}

\author{
Joachim Kaldasch \\ EBC Hochschule Berlin, Alexanderplatz 1, 10178 Berlin, Germany \\ (email: joachim.kaldasch@international-business-school.de)
}

\begin{abstract}
Presented is an evolutionary model of consumer non-durable markets, which is an extension of a previously published paper on consumer durables. The model suggests that the repurchase process is governed by preferential growth. Applying statistical methods it can be shown that in a competitive market the mean price declines according to an exponential law towards a natural price, while the corresponding price distribution is approximately given by a Laplace distribution for independent price decisions of the manufacturers. The sales of individual brands are determined by a replicator dynamics. As a consequence the size distribution of business units is a lognormal distribution, while the growth rates are also given by a Laplace distribution. Moreover products with a higher fitness replace those with a lower fitness according to a logistic law.

Most remarkable is the prediction that the price distribution becomes unstable at market clearing, which is in striking difference to the Walrasian picture in standard microeconomics. The reason for this statement is that competition between products exists only if there is an excess supply, causing a decreasing mean price. When, for example by significant events, demand increases or is equal to supply, competition breaks down and the price exhibits a jump. When this supply shortage is accompanied with an arbitrage for traders, it may even evolve into a speculative bubble. Neglecting the impact of speculation here, the evolutionary model can be linked to a stochastic jump-diffusion model.
\end{abstract}

Keywords: non-durables, evolutionary economics, economic growth, price distribution, Laplace distribution, replicator equation, firm growth, growth rate distribution, competition, jump-diffusion Model 


\section{Introduction}

The evolutionary model of non-durable markets presented here is an extension of a previously published approach to consumer durable goods [1-3]. The key idea is that similar to durable goods, also non-durables must be governed by the evolutionary VariationSelection-Reproduction (VSR) mechanism $[4,5]$. The VSR-mechanism can be understood as follows:

In an anonymous, competitive, non-durable market manufacturers offer the same good with slightly different product features. We want to denote the product variants as brands. We consider firms as consisting of a number of business units producing the corresponding brand. The features are related on the one hand to utility properties of a brand and on the other hand to its product price. There are non-durable goods which are per definition standards (e.g. electric current). In this case the brands differ only in the price.

A business unit can be treated as an input-output system, where the input is the financial revenue and the output is its product supply. Together with the demand side of the market the business units form a reproduction cycle (R). During the reproduction process, however business units vary the features of the brands a bit, in particular the price (V). The preferences of potential consumers determine the selective "environment" for the brands (S). Those brands which correspond closest to consumer demand will be preferentially selected. As a result the reproduction cycle is self-amplifying and characterized by a preferential growth. Specifying the growth rate as the fitness of a brand we will derive below that the unit sales are governed by a replicator dynamics. In evolutionary terms the fittest brands have an evolutionary (competitive) advantage compared to other products. Since brands with a lower fitness have to increase their fitness or disappear from the market, the overall fitness of the good will increase over a long period of time. In other words, the model suggests that Adam Smith "invisible hand" is the evolutionary VSR-mechanism.

Applying statistical methods a model is developed that describes qualitatively the evolution of a non-durable consumer market over a long time period. This model is valid if the product fitness, which is a function of the price, has a single maximum. This maximum is determined by the willingness to pay a minimum price, denoted as the natural price. However, in difference to durables, non-durables are often the subject of speculation. In this case the condition may not be fulfilled, because speculators are interested in an increasing price instead to pay the natural price. They can disturb the market considerably. In order to keep the model simple, the impact of speculators on the market evolution is neglected here.

Within this limitation the time evolution of the sales of the individual brands can be derived from a consideration of demand and supply flows. Also derived is the size and growth rate distribution of the business units (products), and the price distribution and mean price evolution of the brands. Based on empirical investigations, the price evolution for nondurables is usually modelled by stochastic differential equations. In this paper it is shown that the evolutionary model can be mapped qualitatively to a standard jump-diffusion spot price model. 


\section{The Model}

\subsection{The Non-Durable Market}

We start with a consideration of the (static) non-durable market.

\section{The supply side}

The supply side is determined by a number of different variants of the non-durable, denoted as brands, having similar utility properties. They are produced and distributed by manufacturers, while each brand is assigned to a business unit. We want to indicate the brands (and the corresponding business units) with index $i$, while $N>1$ is its total number. (We exclude here a monopoly market). The absolute number of units of the $i$-th brand sold per unit time is denoted $Y_{i}$, while $S_{i}$ indicates the number of supplied units.

The real price $\mu_{i}$ of the $i$-th brand is the nominal price scaled by the mean income. The mean price is determined by:

$$
\langle\mu(t)\rangle=\frac{1}{Y_{t}(t)} \sum_{i=1}^{N} Y_{i}(t) \mu_{i}(t)
$$

while the brackets indicate the average over the sold units, while total unit sales and total supply flow can be obtained from:

$$
Y_{t}=\sum_{i=1}^{N} Y_{i} ; S_{t}=\sum_{i=1}^{N} S_{i}
$$

\section{The demand side}

The demand side can be characterized by an ensemble of agents who are interested in purchasing the non-durable. As for all physical goods the purchase process can be separated into first purchase and repurchase of the good, while repurchase separates into replacement and multiple purchase. In order to understand the first purchase process we have to emphasize that some non-durables are coupled to a complementary durable good (called system coupling). So for example energy is necessary for the application of engines in durable goods (e.g. cars) and therefore first purchase of the corresponding nondurable (e.g. fuel) depends on the diffusion of the durable good. We want to denote the number of agents, who have adopted the durable good as $N(t)$ and the total number of interested agents as the market potential $M$. For inexpensive non-durables products, which have no system coupling (e.g. food) the adoption process $(N(t))$ is limited by the information spreading process, which can be well described by Bass diffusion [1,6,7]. However, the mean lifetime of the non-durable good is short (usually in the order of less than a couple of weeks). Therefore correlations in the unit sales caused by the replacement of the good will, in difference to durable goods, disappear fast. Therefore repurchase can be treated as an effective multiple purchase. Because the first purchase process evolves much slower than the repurchase process, we assume:

i) The repurchase of a non-durable follows the adoption process immediately. 
In this case we can define a total demand rate as:

$D(\langle\mu(t)\rangle)=m q(\langle\mu(t)\rangle) N(t)$

(3)

where $q(\langle\mu>)>0$ is a price dependent multiple purchase rate. It determines the mean number of purchase decisions per adopter per unit time. The demand rate is proportional to the number of adopters, $N(t)$. Note that the demand of the non-durable is determined by the mean amount, per purchase decision $m$. Usually non-durables can be purchase in single units, then $m=1$ unit. But if this is not the case (e.g. fuel) the value of $m$ has to be modified by a corresponding average (e.g. mean tank content).

In order to establish a theory with continuous variables, we scale extensive variables by the market potential $M$, which is a large figure. The corresponding density becomes:

$d(\langle\mu(t)\rangle)=m q(\langle\mu(t)\rangle) n(t)$

(4)

where $n(t)=N(t) / M$. The corresponding purchase and supply flow densities are determined by:

$y_{i}=\frac{Y_{i}}{M} ; s_{i}=\frac{S_{i}}{M}$

The key idea to describe the demand rate is the following assumption:

ii) It exists a minimum mean price $\mu_{m}>0$, at which the demand rate has a maximum.

Following Adam Smith we want to denote this minimum price as the natural price. This assumption reflects the "law" of diminishing marginal utility of a non-durable (Gossen's law). In other words, the willingness to pay has a maximum at $\mu_{m}$ and hence the demand rate in Eq.(4) has a maximum. Sufficiently close to $\mu_{m}$ the total demand rate can be expanded as a function of the mean price as:

$d(\langle\mu\rangle)=d_{m}-\alpha\left(\langle\mu\rangle-\mu_{m}\right)^{2}$

(6)

where $d_{m}$ is a maximum demand. For some non-durables $d_{m}$ exhibits periodic variations (e.g. electricity). Note that as mentioned above the impact of speculation is neglected.

\subsection{The Market Dynamics}

The market dynamics is determined by both, the supply and demand side. 


\section{The supply side}

The key process on the supply side is the production and distribution of the good by the business units (firms). As discussed above we can consider the business units as inputoutput systems, where the financial flow (revenue) is the input and the output is the product supply. The supply flow can therefore be viewed as a function of the unit sales $s_{i}=s_{i}\left(y_{i}\right)$. Expanding the supply of the $i$-th brand up to the first order we obtain:

$s_{i}\left(y_{i}, t+\delta t\right) \cong c_{1}+c_{2} y_{i}(t)$

where $\delta t$ is a short time increment indicating the response time on an input variation. In order to keep the model simple this time shift is neglected, $\delta t=0$.

Without input flow (proportional to the unit sales $y_{i}$ ) the output flow $s_{i}$ will be zero, hence $c_{1}=0$. Further we write $c_{2}=1+\gamma_{i}(t)$, where the productivity $\gamma(t)$ is denoted as reproduction coefficient. We obtain for the supply flow:

$s_{i}(t)=\left[1+\gamma_{i}(t)\right] y_{i}(t)$

This relation determines that the supply side completely. It suggests that the supply of a brand follows the evolution of the unit sales. Differences between unit sales and supply flow are contained in the reproduction coefficient. The total supply obeys the relation:

$s_{t}(t)=[1+\langle\gamma(t)\rangle] y_{t}(t)$

while $\langle\gamma\rangle$ is the mean reproduction coefficient.

\section{The demand side}

The key idea to determine the demand side is to consider the repurchase process as consisting of statistical events, where potential consumers meet available units of the $i$-th brand (e.g. in stores, the internet etc.) and purchase it with a certain probability. In order to establish a relation for the unit sales of the $i$-th brand, we introducing the density of potential consumers $\psi$, as the number of potential consumers scaled by the market potential. Further the density of available unit's $x_{i}$, is the total number of units available in stores scaled by the market potential. The unit sales $y_{i}$ must be zero if there are either no potential consumers or available units. Expanding the unit sales of the $i$-th brand up to the first order, $y_{i}$ must be proportional to the product of both densities. Hence purchase events occur with a frequency:

$y_{i} \cong \eta_{i} x_{i} \psi\left(\mu_{i}\right)$

where the probability rate $\eta_{i}>0$ specifies the mean success of the $i$-th model and is denoted as the preference parameter. This parameter characterizes the product features and the spatial distribution of a brand. The product price $\mu_{i}$ for which the brand is available limits the density of potential consumers $\psi\left(\mu_{i}\right)$. Note that Eq.(10) expresses Say's theorem, which suggests that supply creates its own demand. 
The densities of potential consumers and available units can be obtained from balance relations (conservation laws). The density of available products of a brand, is determined by the balance between supply and purchase flow:

$\frac{d x_{i}}{d t}=s_{i}-y_{i}=\gamma_{i} y_{i}$

where we used Eq.(8). That means the density of available unit's increase, when there is a higher supply flow than purchase flow proportional to the reproduction parameter.

The density of potential consumers is governed on the other hand by the generation of new potential adopters given by the demand rate, and their disappearance due to the purchase of the good:

$$
\frac{d \psi}{d t}=d-y_{t}
$$

The stationary density of potential consumers $\psi_{S}$ is determined by the condition $\mathrm{d} \psi / d t=0$. In this state the demand rate is just equal to the sales:

$d(\langle\mu(t)\rangle)=y_{t}(\langle\mu(t)\rangle)$

Remarkable is that, despite from small fluctuations, this relation always holds, as long as the demand rate is just a function of the mean price. This can be seen by testing the stability of the stationary density $\psi_{S}$. Perturbing this state by a small quantity $\delta \psi(\tau)$, such that $\psi(\tau)=\psi_{S}+\delta \psi(\tau)$ and inserting this relation into Eq.(12) we get with Eq.(10):

$\frac{d \delta \psi}{d t}=-\delta \psi \sum \eta_{i} x_{i}$

Writing a time dependent fluctuation as:

$\delta \psi(t) \sim e^{\lambda t}$

we obtain that $\lambda$ is negative, because $\sum \eta_{i} x_{i}>0$. Therefore, small fluctuations always disappear and $\psi_{S}$ is a stable state for a given mean price. For the stationary state the density of potential adopters becomes:

$\psi_{S}(\langle\mu\rangle)=\frac{1}{\sum \eta_{i} x_{i}} d(\langle\mu\rangle)=\psi_{0} d(\langle\mu\rangle)$ 
Obviously Eq.(13) suggests that the total unit sales can be obtained from the total demand using Eq. (6), for a given mean price. Therefore we have to focus on the mean price evolution. The key idea to specify the mean price evolution is the assumption:

iii) In a competitive market the mean price varies much slower than the price of the individual brands.

This assumption is called in standard microeconomics price rigidity [8]. It allows us to introduce a sufficiently short time scale $\tau$ on which the mean price can be considered to be constant. The short is related to the long time scale according to:

$t=\varepsilon \tau$

with $\varepsilon<<1$. (For example if $t$ is in years and $\tau$ in weeks, $\varepsilon \approx 1 / 50$ ). On this time scale the mean price is a constant:

$\frac{d\langle\mu\rangle}{d \tau} \approx 0$

However, if the mean price is a constant on the short time scale, Eq.(13) suggests that also the total demand and the corresponding total sales must be a constant:

$\frac{d y_{t}}{d \tau} \approx 0$

This result implies that all brands together cannot sell more than $y_{t}$, which leads to a considerable competition between the brands. The individual unit sales can be obtained on the short time scale from a time derivative of Eq.(10):

$\frac{d y_{i}(\tau)}{d \tau}=x_{i} \psi_{S}\left(\mu_{i}\right) \frac{d \eta_{i}(\tau)}{d \tau}+\eta_{i} x_{i} \frac{d \psi_{S}\left(\mu_{i}\right)}{d \tau}+\eta_{i} \psi_{S}\left(\mu_{i}\right) \frac{d x_{i}(\tau)}{d \tau}$

while we have used that the total market is in a stationary state. We can consider price and preference (utility) variations as constant interrupted by small jumps on the short time scale. The first two summands can therefore be treated to contribute additive fluctuations $\delta(\eta, \mu)$. Taking advantage of Eq.(11), we obtain for the third term:

$\frac{d y_{i}(\tau)}{d \tau}=\eta_{i} \psi_{S}\left(\mu_{i}\right) \gamma_{i} y_{i}+\delta\left(\eta_{i}, \mu_{i}\right)$

This relation suggests that price and preference jumps induce multiplicative fluctuations of the unit sales. The multiplicative fluctuations will have a higher impact on the sales evolution than additive fluctuations.

Neglecting the latter and introducing the rate: 
$f_{i}=\eta_{i} \gamma_{i} \psi_{S}\left(\mu_{i}\right)$

(22)

Eq.(21) turns into

$\frac{d y_{i}(\tau)}{d \tau}=f_{i} y_{i}(\tau)$

(23)

The constraint Eq.(19), can be satisfied by adding a constant growth rate $\zeta$ such that:

$\frac{d y_{i}(\tau)}{d \tau}=\left(f_{i}-\zeta\right) y_{i}(\tau)$

(24)

Inserting this relation into Eq.(19) we obtain:

$\zeta=\langle f\rangle=\frac{\sum_{i} y_{i} f_{i}}{y_{t}}$

Rewriting Eq.(24), the sales evolution of the $i$-th brand is determined by the replicator equation:

$\frac{d y_{i}(\tau)}{d \tau}=\left(f_{i}-\langle f\rangle\right) y_{i}(\tau)=r_{i} y_{i}(\tau)$

(26)

where we have introduced the growth rate of the $i$-th brand in terms of unit sales, $r_{i}$. Because from evolutionary models the parameter $f_{i}$ in the replicator equation is known as the fitness, we want to denote $f_{i}$ here as the product fitness.

This is a remarkable result, because it suggests that the brands stand in an evolutionary competition. The sales of those brands with a higher product fitness than the mean fitness increase. The fitness space is determined by the preference parameter $\eta$, the demand rate as a function of the product price $\mu$ and the reproduction coefficient $\gamma$. The fitness has therefore contributions from both sides of the market. (Note that strictly speaking

Due to random price jumps, the fitness fluctuates and the unit sales of a business unit are governed by a stochastic multiplicative process. The size distribution of the business units $P_{y}$, which is determined by the probability to find the unit sales of a business unit $y_{i}$ in the interval $y$ and $y+d y$, can be derived for the case that $f_{i}$ can be treated as an independent, identical distributed, random variable. The central limit theorem suggests that the size distribution of the business units is given for a sufficiently long time by a lognormal probability distribution function (pdf) of the form: 
$P_{y}(y, t)=\frac{1}{\sqrt{2 \pi t} \omega y} \exp \left(-\frac{\left(\ln \left(y / y_{0}\right)-u t\right)^{2}}{2 \omega^{2} t}\right)$

where $u$ and $\omega$ are free parameters and $y / y_{0}$ is the size of the business unit scaled by the size at $t=0$.

In our further considerations we want to consider the brands as localized in the fitness space. We write the fitness parameters as the sum of a mean value indicated by the brackets and small variations:

$\mu_{i}=\left(\langle\mu\rangle+\Delta \mu_{i}\right)$

$\eta_{i}=\left(\langle\eta\rangle+\Delta \eta_{i}\right)$

and

$\gamma_{i}=\left(\langle\gamma\rangle+\Delta \gamma_{i}\right)$

(30)

The mean price evolution

For the consideration of the price evolution of a non-durable, we want to treat the impact of variations of the reproduction parameter and the preference as small and neglect the fluctuations $\Delta \gamma$ and $\Delta \eta$. Further we introduce the price distribution $P_{\mu}$, which is determined by the relative abundance that a product is purchased in the price interval $\mu$ and $\mu+d \mu$. The price distribution has its mean at:

$$
\langle\mu\rangle=\int_{0}^{\infty} P_{\mu}\left(\mu^{\prime}\right) \mu^{\prime} d \mu^{\prime}
$$

and a variance:

$\operatorname{Var}\left(P_{\mu}\right)=\left\langle\left(\mu_{i}-\langle\mu\rangle\right)^{2}\right\rangle$

With Eq.(28) the fitness of the $i$-th brand can be expanded as:

$f\left(\mu_{i}(\tau)\right)=f(\langle\mu(t)\rangle)+\frac{d f(\langle\mu(t)\rangle)}{d \mu} \Delta \mu_{i}(\tau)$ 
The price evolution of the brands can be separated into the evolution of the mean price and its price deviations:

$\frac{d \mu_{i}}{d \tau}=\frac{d \Delta \mu_{i}}{d \tau}+\frac{d\langle\mu(t)\rangle}{d \tau} \cong \frac{d \Delta \mu_{i}}{d \tau}$

Because the mean price varies slowly the price distribution undergoes a fast relation to its stationary state.

The long term evolution of the mean price is determined by the evolution of the mean fitness. Using Eq.(31) we obtain $f(\langle\mu>)=\langle f\rangle$. Taking the time derivative, the mean fitness evolves as:

$\frac{d\langle f\rangle}{d t}=\frac{d f(\langle\mu\rangle)}{d \mu} \frac{d\langle\mu\rangle}{d t}$

Because the mean fitness is determined by the demand rate Eq.(16) :

$\frac{d f(\langle\mu\rangle)}{d \mu} \sim \frac{d d(\langle\mu\rangle)}{d \mu} \sim\left(\langle\mu\rangle-\mu_{m}\right)$

the mean fitness has its stationary state $(d\langle f\rangle d t=0)$ when the mean price is equal to the natural price. Derived in Appendix A is the evolution of the mean price. The mutual competition between the brands causes a mean price evolution of the form:

$\langle\mu(t)\rangle=\mu_{0} e^{-a t}+\mu_{m}$

where $\mu_{0}$ is the price at an initial time $t=0$, and the parameter:

$a=\varepsilon\langle\eta\rangle\langle\gamma\rangle \psi_{0} \alpha \operatorname{Var}\left(P_{\mu}\right)$

is denoted as a price decline rate. Note that the decline rate is zero for a monopoly market, because $\operatorname{Var}\left(P_{\mu}\right)=0$. The price decline is therefore a direct consequence of the competition between the brands, while the variance is treated here as a constant.

The price distribution $P_{\mu}$ can be determined from the following assumption: 
iv) When product sales increase considerably, the business units respond by increasing the price and vice versa.

This assumption takes into account the fact that the manufacturers have finite production capacities on the short time scale. On the one hand they want to use their capacities, but on the other hand they have an upper capacity limit. The business units, however, respond only on the direction of sales variations. That means, variations of the price are proportional to the sign of a sales variation of the $i$-th brand. This can be formulated as follows:

$\frac{d \mu_{i}}{d \tau}=\frac{d \Delta \mu_{i}}{d \tau} \sim \operatorname{sign}\left(\frac{d y_{i}}{d \tau}\right)$

because the mean price is a constant on this time scale. This relation still holds, if we scale within the sign-function by the positive variable $y_{i}$. Because the sales are governed by the replicator equation and we can write:

$$
\frac{d \Delta \mu_{i}}{d \tau} \sim \operatorname{sign}\left(\frac{1}{y_{i}} \frac{d y_{i}}{d \tau}\right) \sim \operatorname{sign}\left(f\left(\mu_{i}\right)-\langle f\rangle\right)
$$

The present theory therefore suggests that price variations are caused by fitness fluctuations of the products around the mean fitness. Taking advantage from the expansion Eq.(31) the relation can be rewritten as:

$$
\frac{d \Delta \mu_{i}}{d \tau} \sim \operatorname{sign}\left(\frac{d f(\langle\mu\rangle)}{d \mu} \Delta \mu_{i}\right)
$$

We have to emphasize that the parameters $\langle\mu\rangle$ and $\langle\eta\rangle$ are always positive for brands with non-zero unit sales. The mean reproduction parameter, however, is governed by Eq.(9):

$$
\langle\gamma(t)\rangle=\frac{s_{t}(\langle\mu(t)\rangle)}{d(\langle\mu(t)\rangle)}-1
$$

where we used Eq.(13). The reproduction parameter is not strictly positive. If total supply flow is less than the total demand rate, the mean reproduction parameter becomes negative.

Therefore we have to distinguish between two cases:

A) The stable regime: $\langle\gamma\rangle>0$.

In this case is a $>0$ and the mean price declines with time. Further is: 
$\frac{d f(\langle\mu\rangle)}{d \mu} \sim \frac{d d(\langle\mu\rangle)}{d \mu}<0$

Therefore Eq. (40) suggests that price differences disappear. This can be interpreted as if there is a restoring force $F(\Delta \mu)$ that drives the price of the individual brands back to the mean price with:

$F(\Delta \mu) \sim-\operatorname{sign}(\Delta \mu)$

The restoring force has its origin the excess supply and the resulting competition between the brands. Writing the restoring force as consisting of a deterministic part given by Eq.(42) and a stochastic contribution $\delta \mu$, Eq.(39) turns into a stochastic Langevin equation:

$\frac{d \Delta \mu}{d \tau}=F(\Delta \mu)+\delta \mu(t)$

For the simplifying assumption that the random price variations are uncorrelated, the fluctuations can be treated as white noise with mean value und time correlation:

$$
\begin{aligned}
& \langle\delta \mu(\tau)\rangle_{\tau}=0 \\
& \left\langle\delta \mu(\tau), \delta \mu\left(\tau^{\prime}\right)\right\rangle_{\tau}=D \delta\left(\tau-\tau^{\prime}\right)
\end{aligned}
$$

where the brackets with index $\tau$ indicate the time average and $D$ is a noise amplitude. Based on the corresponding Fokker-Planck equation, the stationary price distribution can be obtained (Appendix B):

$$
P_{\mu}(\Delta \mu) \sim \exp \left(-\frac{2 b}{D}|\Delta \mu|\right)
$$

where $b=\langle\Delta \mu\rangle_{\tau} / \Delta \tau$ is a time average over the response of the business units on sales variations. The price distribution in the case of a competitive market is therefore approximately given by a Laplace (double exponential) distribution. In a semi-log plot, the Laplace distribution has a tent shape around the mean price, $\langle\mu(t)\rangle$.

B) The unstable regime: $\langle\gamma\rangle \leq 0$.

In this case is $a<0$ and the mean price increases. Because:

$$
\frac{d f(\langle\mu\rangle)}{d \mu} \geq 0
$$


there is no restoring force for the price distribution and the price distribution becomes unstable, because all manufacturers experience capacity limitation. In difference to standard microeconomics the theory therefore suggests that at market clearing the price becomes unstable. It goes beyond the abilities of the model to describe the unstable regime exactly. Though, we can expect that the condition of a slowly varying mean price is not fulfilled (on the chosen short time scale). In the unstable regime and the mean price will therefore exhibit a rapid increase.

The model therefore suggests that the time evolution of the mean price of a nondurable market separates into two regimes, a stable and an unstable regime. This result of the model is displayed in Fig. 1 for a storable non-durable good. Shown is the demand rate $d(\mu)$ and supply curve $s(\mu)$. Eq.(40) suggests that the stable regime is characterized by an excess supply $\langle\gamma\rangle>0$. Here total supply $s_{t}$ increases total demand (unit sales) $y_{t}$ and the excess supply $x_{t}$ is stored and available for consumers to purchase. The excess supply induces competition between the brands and the price fluctuates around the mean price according to a Laplace distribution. As a consequence of this competition the price declines on the long time scale according to an exponential law approaching the minimum price $\mu_{m}$ (Eq.(35)).

The price can increase for non-durables either slowly or rapidly. A shallow price increase occurs if the total demand increases faster in time than total supply. This can be the case when the number of adopters $N(t)$ increases in time, which is usually a slow process. A rapid increase of the price, however, has its origin in a supply shortage cause for example by a rare event (e.g. weather events, catastrophes etc.). In this case total demand increases or is equal to total supply, $\langle\gamma\rangle \leq 0$, and manufacturers can get rid all of their products (Fig.1). Because there is no competition the price distribution is unstable in this regime, and causes a rapid price increase. Theoretically, these price jumps should be small because a fluctuation is usually close to $\langle\gamma\rangle=0$, and ends in the stable regime where $\langle\gamma\rangle>0$.

However, a rapid price increase may change the purchase behaviour of the consumers for non-durables. In expecting a further rising price, they start to store the non-durable, which can be interpreted as an increased preference. As a result the demand curve shifts effectively such that the distance to $\langle\gamma\rangle=0$ increases, and the market remains even longer in the unstable regime. This is a case of self-fulfilling prophecy. In addition the price increase offers traders an extra arbitrage. Therefore speculators start to purchase additional amounts of the nondurable. When this happens, the supply shortage turns into a speculative bubble and the jump size of the price increase can hardly be predicted.

Arrived in the stable regime the price starts in the long term to decrease, due to mutual competition between the brands. If the impact of speculations is small, the mean price decreases on average according to an exponential law. However, if a considerable amount of the non-durable is stored for speculative reasons, the traders are forced to sell the good in order to make their arbitrage. This behaviour of the traders leads to considerable price jumps. Because we have neglected in this model the impact of speculators, displayed in the insert of Fig. 1 is the price evolution after a supply shortage as can be expected from the evolutionary model. After a price increase the price relaxes towards $\mu_{m}$. But the mean price cannot come deep into the unstable region. The price approaches and repels from $\mu_{c}(t)$ and has stable periods intermitted by unstable phases, if $\mu_{c}(t)>>\mu_{m}$.

\section{Mapping the evolutionary to a stochastic model}

From the study of the empirical time evolution stochastic spot price models where established for non-durables, having its origins in the investigation of financial markets [9]. 
We want to link the evolutionary dynamics derived above to standard stochastic spot price dynamics.

The simplest model taking into account mean-reverting behavior in a stochastic approach is given by an Ornstein-Uhlenbeck process (Vasicek model) [10]. Here the price evolution is a diffusion process satisfying the stochastic differential equation:

$d \mu=-\lambda \Delta \mu d \tau+\sigma d W$

where $W$ is a standard Brownian motion (Wiener process), $\sigma$ the constant volatility of the process and $\lambda$ the velocity with which the price reverts on the short time scale to its long term mean price $\langle\mu(t)>$. This relation corresponds to Eq.(43) in the evolutionary model. However, the fluctuations are governed for large price deviations by a fat tailed Laplace distribution, not by a normal distribution. Note that fat tails can be also modeled by setting the volatility $\sigma$ proportional to $\mu$, which was done for example in the Pilipovic model [11].

Significant but rare events may lead to a supply shortage. In this case the evolutionary price distribution becomes unstable causing a rapid increasing price. These price jumps can be included in stochastic differential equations by introducing additional jump terms [12]. The evolutionary model can therefore be mapped to a jump-diffusion stochastic equation of the general form:

$d \mu=\lambda(\mu, \tau) d \tau+\sigma(\mu, \tau) d W+J(\mu) d Q(f)$

where the arrival of jumps is usually treated as a Poisson process $d Q(f)$ with arrival frequency parameter $f$ and jump size $J$. Several models were developed for the price evolution including jump-diffusion models with appropriate functions $\lambda, \sigma$ and $J[9,13]$.

While on the short time scale $\langle\mu(t)\rangle$ can be treated as a constant, on the long time scale the mean price decreases exponentially towards $\mu_{m}$ (with eventual seasonal variations), approaching the price at market clearing $\mu_{c}(t)(\langle\gamma\rangle=0)$. The exponential decline can be interpreted as a mean reverting process equivalent to Eq.(47). However, market clearing depends on variations in the intersection of the supply and demand curve. Therefore, $\mu_{c}(t)$ varies on the long time scale and can be treated also as a fluctuating variable (Pilipovic model).

The growth rate distribution

The growth rate distribution $P_{r}(r)$ determines the relative abundance of a business unit to have a growth rate in the interval $r$ and $r+d r$. Because the growth rate and the price variations are related by $\Delta \mu=(d f / d \mu)^{-1} r$, the short term growth rate distribution can be obtained by changing the variables in Eq.(45). Therefore the growth rate distribution is also determined by a Laplace distribution:

$P_{r}(r) \sim \exp (-|r|)$ 


\section{A constant fitness advantage}

We want to discuss the impact of a constant fitness advantage over a long time period. The market can be reduced to the two product case, $i=1,2$. The first product has fitness $f_{1}$, while all other brands can be summarized to the second product with fitness $f_{2}$, such that $f_{1}>>f_{2}$. Applying the quotient rule to the replicator equation we obtain:

$\frac{d}{d \tau}\left(\frac{y_{1}}{y_{2}}\right)=\frac{1}{y_{2}^{2}}\left[\frac{d y_{1}}{d \tau} y_{2}-\frac{d y_{2}}{d \tau} y_{1}\right]=\left(\frac{y_{1}}{y_{2}}\right) \theta$

where the fitness advantage is:

$\theta=f_{1}-f_{2}$

The differential equation can be solved by a separation of the variables. Taking advantage from the assumption that $\theta$ is a constant, the time evolution of the market shares on the long time scale has the form:

$\ln \left(\frac{y_{1}}{y_{2}}\right)=\theta \varepsilon t+C_{m}$

while $C_{m}$ is an integration constant. This result suggests that the relation $y_{1} / y_{2}$ respectively the corresponding market shares must be a linear function plotted in a half- logarithmic diagram. We can conclude that a constant fitness advantage $\theta$, leads to a replacement of the current products over a long time period according to a logistic law.

\section{Conclusion}

Presented is an evolutionary model for non-durable markets, which is equivalent to a model for durable goods, taking the specifics of non-durables into account. Their key features are a short lifetime and usually low costs for a single unit. At introduction a brand faces the competition with other brands and must therefore have at least an equivalent or higher fitness than the other brands. In the introduction phase the market penetration of a non-durable can be expected to be governed by Bass diffusion. However, when a non-durable forms a system with a durable good, its penetration evolves parallel to the diffusion of the durable good. This paper focuses not on the market penetration, but on the sales and price dynamics of nondurable goods. The model suggests that similar to durables the dynamics of the individual non-durable brands is governed by a replicator equation in a competitive market.

Note that for consumer durables, a supply shortage is rather unlikely because due to its long lifetime a repurchase of the good can be shifted. This condition is usually not fulfilled for non-durables. Owing the short lifetime, non-durables must be permanently repurchased. Therefore in difference to durables a supply shortage may occur and we have to take the possibility into account that total demand increases total supply. From the evolutionary point of view this is a dramatic event, because when all producers can sell the entire output, competition brakes down. The evolutionary model suggests that in this case the price 
distribution becomes unstable at market clearing, associated with a rapid price increase. Therefore the price-demand/supply diagram separates into a stable and an unstable region at market clearing (Fig.1). Theoretically the resulting price jumps should be usually small, because a slightly increasing mean price leads usually to an excess supply, which reestablishes competition. (Note that in the electricity market, because the non-durable can hardly be stored, day-ahead auctions lead to a market clearing of produced and supplied current defining a spot price. However, the competition in this market takes place not with delivered current, but with future capacities. A market clearing as meant in this model occurs when all available potential capacities equals total future demand. Approaching the clearing point, however, the inelastic demand climes up rapidly the increasing supply curve. This leads to a spot price jump even before capacity clearing. When capacity clearing occurs, avoiding a blackout, a country is forced to import additional capacities.)

However, when the consumer behaviour changes upon this event (by an increased preference) and moreover traders try to earn an extra arbitrage, the supply shortage may turn into a speculative bubble. The chance that durables suffer form speculation is rather low, because there is usually an excess supply of durables. But economic history shows that even durables can create speculative bubbles. This is particular the case for the real estates [14].

As long as the impact of speculation can be neglected the price reverts exponentially and approaches the natural price asymptotically. However, if there is a considerable amount of the good purchased for speculative reasons, the price may exhibit rapid jumps. This behaviour is known for example from the oil price evolution. The present model suggests that the dramatic price variations are rather caused by speculators and not by a real demand. There are two ways to take speculation into account. Either speculation is included into the total demand curve or as an extra dynamics. Including speculation to demand leads to a multiple intersection of the demand and supply curve and to the assumption of a backward bending supply curve $[15,16]$. However, it can be expected that speculation introduces an extra dynamics, because traders have to respond not only on the price, but also on price variations.

The presented evolutionary model neglects the impact of a speculative component. While for durables this condition is usually fulfilled the model is limited only to those nondurables satisfying this constrained. In this case the short term price evolution can be mapped to a stochastic jump-diffusion model, applied among other models to non-durables [13]. The predicted fat tailed price distribution can be found for example in empirical investigations of the electricity spot price [17].

Finally we want to summarize some key differences of the presented model to neoclassic microeconomics, emphasizing the evolutionary economic perspective [18].

1. The microeconomic approach neglects preference differences between brands of the same good, while the evolutionary model takes them explicitly into account.

2. Standard microeconomics derives total demand from utility considerations under budget constraints. However, utility is not an absolute variable. The utility in terms of product features is contained in the evolutionary model in the preference parameter. Total demand is related in the present model to the assumption that at natural price, the demand rate must have a maximum (Gossen's law).

3. The evolutionary model treats the purchase process as statistical events (Say's theorem), and the dynamics is governed by balance equations.

4. The dynamics of the supply side is determined by the reproduction cycle. Generally supply follows demand, while deviations are contained in a reproduction parameter.

5. Because the mean price evolves slowly, known in standard microeconomics as price rigidities, the total demand rate also evolves slowly. When total supply increases total demand the brands suffer from mutual competition. This is the key point of the model! If there is no extra supply, competition brakes down. 
6. In a competitive market the unit sales are governed by a replicator dynamics. The individual brands can be characterized by product fitness, depending on the price, preference and reproduction parameter. The replicator dynamics leads to an exponential decline of the mean price towards the natural price.

7. Taking into account that the manufacturers want to use their limited capacities, their behaviour leads to a Laplace price distribution if their price decisions can be treated as uncorrelated. The price fluctuations are initiated by fitness fluctuations of the brand.

8. Products with a higher fitness replace those with a lower fitness according to a logistic law. The good can be considered to evolve over a long period of time trough a series of replacements, improving the overall fitness $[19,20]$.

9. The size distribution of the business units (in terms of unit sales) must be lognormal, while their growth rate is approximately determined by a Laplace distribution.

10. Competition brakes down and the market becomes unstable at market clearing (Fig.1). This is the most striking difference to the Walrasian picture, which suggests that demand equals supply.

11. When demand is equal or increases supply, caused for example by a significant event, the price distribution becomes unstable, i.e. there is no restoring force towards mean price. As a result the price exhibits a rapid increase. But only if speculation comes into play, this price jump can evolve into a speculative bubble. When the price is close to market clearing it undergoes periods of stability intermitted by unstable events. This was suggested by Mandelbrot to be a general property for markets [21]. The cobweb model is incorrect.

12. In standard microeconomics, agents have to maximize their utility respectively their profit under constraints. This maximizing process takes place in the evolutionary model by maximizing the product fitness. However, in this model economic agents do not have to calculate its marginal utility. The optimization takes place on the basis: success breads success (VSR mechanism).

13. Note that the model is not applicable to monopolies. Monopolies are just limited by the product demand for a given price [2].

Evolutionary processes come into play only statistically. Therefore this theory suffers from the problem to establish a direct relation to the individual behaviour of consumers and manufacturers. The situation is similar to statistical mechanics, where thermodynamic relations are the result of a statistical average and the individual motion has only a probalistic meaning. Therefore the evolutionary model is limited to sufficiently large markets, respectively market segments. 


\section{Appendix A}

\section{The Mean Price Evolution}

In this chapter we want to consider the evolution of the mean price. We take advantage from Eq.(33) and write:

$$
\frac{d\langle\mu\rangle}{d t}=\varepsilon\left(\frac{d f(\langle\mu\rangle)}{d \mu}\right)^{-1} \frac{d\langle f\rangle}{d t}
$$

where

$$
\frac{d f(\langle\mu\rangle)}{d \mu}=\langle\eta\rangle\langle\gamma\rangle \psi_{0} \frac{d d(\langle\mu\rangle)}{d \mu}=-2\langle\eta\rangle\langle\gamma\rangle \psi_{0} \alpha\left(\langle\mu\rangle-\mu_{m}\right)
$$

using Eq.(6). The time derivative of the mean fitness can be found by using the quotient rule:

$$
\frac{d\langle f\rangle}{d t}=\frac{d}{d t} \sum_{i} f_{i} \frac{y_{i}}{y_{t}}=\frac{y_{t} \sum_{i} f_{i}\left(d y_{i} / d t\right)-\sum_{i} y_{i} f_{i}\left(d y_{t} / d t\right)}{y_{t}{ }^{2}}=\frac{\sum_{i} f_{i}\left(f_{i} y_{i}\right)}{y_{t}}-\langle f\rangle^{2}=\sigma_{f}{ }^{2}
$$

where

$$
\sigma_{f}^{2}=\sum_{i} \frac{y_{i}}{y_{t}}\left(f_{i}-\langle f\rangle\right)^{2}=\sum_{i} \frac{y_{i}}{y_{t}}\left(f_{i}\right)^{2}-\langle f\rangle^{2}
$$

is the variance of the fitness. Applying the expansion Eq.(31) we further obtain:

$$
\sigma_{f}{ }^{2}=2\langle f\rangle \frac{d f(\langle\mu\rangle)}{d \mu} \sum_{i} \frac{y_{i}}{y_{t}} \Delta \mu_{i}+\left(\frac{d f(\langle\mu\rangle)}{d \mu}\right)^{2} \sum_{i} \frac{y_{i} \Delta \mu_{i}^{2}}{y_{t}}=\left(\frac{d f(\langle\mu\rangle)}{d \mu}\right)^{2} \sigma_{\mu}{ }^{2}
$$

where we used that $\langle f\rangle=f(\langle\mu\rangle)$ and $\langle\delta \mu>=0$. The price variance is defined as:

$$
\operatorname{Var}\left(P_{\mu}\right)=\sigma_{\mu}{ }^{2}=\sum_{i} \frac{y_{i}}{y_{t}}\left(\Delta \mu_{i}-\langle\mu\rangle\right)^{2}
$$

Hence we get:

$$
\frac{d\langle\mu\rangle}{d t}=\varepsilon \frac{d f(\langle\mu\rangle)}{d \mu} \operatorname{Var}\left(P_{\mu}\right)
$$


(A7)

which is a general result, caused by the competition between brands [1]. With Eq.(A2), the price evolution close to $\mu_{\mathrm{m}}$ is governed by:

$\frac{d\langle\mu(t)\rangle}{d t}=-2 \varepsilon\langle\eta\rangle\langle\gamma\rangle \psi_{0} \alpha \operatorname{Var}\left(P_{\mu}\right)\left(\langle\mu(t)\rangle-\mu_{m}\right)$

(A8)

For constant variables the time evolution on the long time scale, is determined by:

$\langle\mu(t)\rangle=\mu_{0} e^{-a t}+\mu_{m}$

(A9)

where $\mu_{0}$ is an integration constant and the parameter

$a=\varepsilon\langle\eta\rangle\langle\gamma\rangle \psi_{0} \alpha \operatorname{Var}\left(P_{\mu}\right)$

(A10)

is denoted as the price decline rate. This result is equivalent to the case of a competitive consumer durable market [1,2]. 


\section{Appendix B}

The corresponding Fokker-Planck equation of the Langevin equation (43) for the price probability distribution can be written as:

$\frac{\partial P_{\mu}(\Delta \mu, t)}{\partial t} \sim \frac{\partial}{\partial \mu}\left(-F(\Delta \mu) P_{\mu}(\Delta \mu, t)+\frac{1}{2} D \frac{\partial P_{\mu}(\Delta \mu, t)}{\partial \mu}\right)$

(B1)

For a sufficient long time the probability distribution approaches its stationary state determined by:

$P_{\mu}(\Delta \mu) \sim \exp \left(-\frac{2}{D} \Omega(\Delta \mu)\right)$

with the generalized potential:

$\Omega(\Delta \mu)=-\int F\left(\Delta \mu^{\prime}\right) d \Delta \mu^{\prime}$

(B3)

The integration can be carried out. With Eq.(42) for the restoring force we obtain for the stationary price distribution:

$P_{\mu}(\Delta \mu)=\frac{b}{D} \exp \left(-\frac{2 b}{D}|\Delta \mu|\right)$

The price distribution is therefore given by a Laplace (double exponential) distribution. The variance of the price distribution is time independent and given by:

$\operatorname{Var}\left(P_{\mu}\right)=\frac{1}{2} \frac{D^{2}}{b^{2}}$

(B5)

for a constant $b=\langle\Delta \mu\rangle_{\tau} / \Delta \tau$, which is a time average over the response of the business units on sales variations. 


\section{References}

[1] J. Kaldasch, Evolutionary Model of an Anonymous Consumer Durable Market, Physica A, 390, (2011) 2692-2715.

[2] J. Kaldasch, The Product Life Cycle of Durable Goods, Preprint condmat/11090828 (2011).

[3] J. Kaldasch, The Experience Curve and the Market Size of Competitive Consumer Durable Markets, Preprint MPRA Paper/33370 (2011).

[4] E.D. Beinhocker, The Origin of Wealth, Harvard Business School Press (2006).

[5] R. Feistel, W. Ebeling, Physics of Self-Organization and Evolution, Wiley VCH (2011).

[6] E. M. Rogers, Diffusion of Innovations, eds. Simon and Schuster, New York, (2003).

[7] F. M. Bass, A new product growth model for consumer durables, Management Science 15 (1969) 215-227.

[8] H.R. Varian, Intermediate Microeconomics - A Modern Approach W.W. Northern \& Company Inc. New York (2006).

[9] R.N. Mantegna, E.H. Stanley, An Introduction to Econophysics, Correlations and Complexity in Finance. Cambridge University Press (2007).

[10] O. Vasicek, An Equilibrium Characterization of the Term Structure, Journal of Financial Economics, 5, (1977)177 - 188.

[11] D. Pilipovic, Energy Risk: Valuing and Managing Energy Derivatives. McGraw-Hill (1997).

[12] B. Øksendal, Stochastic Differential Equations: An Introduction with Applications, Springer, New York (1998).

[13] H. Geman, Commodities and Commodity Derivatives: Modelling and Pricing for Agriculturals, Metals, and Energy. John Wiley \& Sons Ltd. (2005).

[14] L. Tvede, Business Cycles, History, Theory and Investment Reality, Jon Wiley \& Sons (2006).

[15] J. Cremer, D. Salehi-Isfahani, Models of the Oil Market. Hardwood Academic Publishers (1991).

[16] S. Jäger, Nonlinear and Stochastic Dynamical Systems Modeling Price Dynamics, Dissertation Thesis, Bonn (2008).

[17] G. Bottazzi, S. Sapio, A Secchi, Some Statistical Investigations on the Nature and Dynamics of Electricity Prices, LEM Working Paper Series 13 (2004).

[18] K. Dopfer, The Evolutionary Foundation of Economics. University Press Cambridge UK, (2005).

[19] C. Marchetti, Primary Energy Substitution Models: On the Integration between Energy and Society, Technological Forecasting and Social Change, 10 (1977) 345-356.

[20] N.M. Victor, J.H. Ausubel, DRAMs as Model Organisms for Study of Technological Evolution, Technological Forecasting and Social Change, 69 (2002) 243-262.

[21] B.B. Mandelbrot, R.L. Hudson, The (Mis) Behaviour of Markets: A Fractal View of Risk, Ruin and Reward. Profile Books (2005). 
Figures

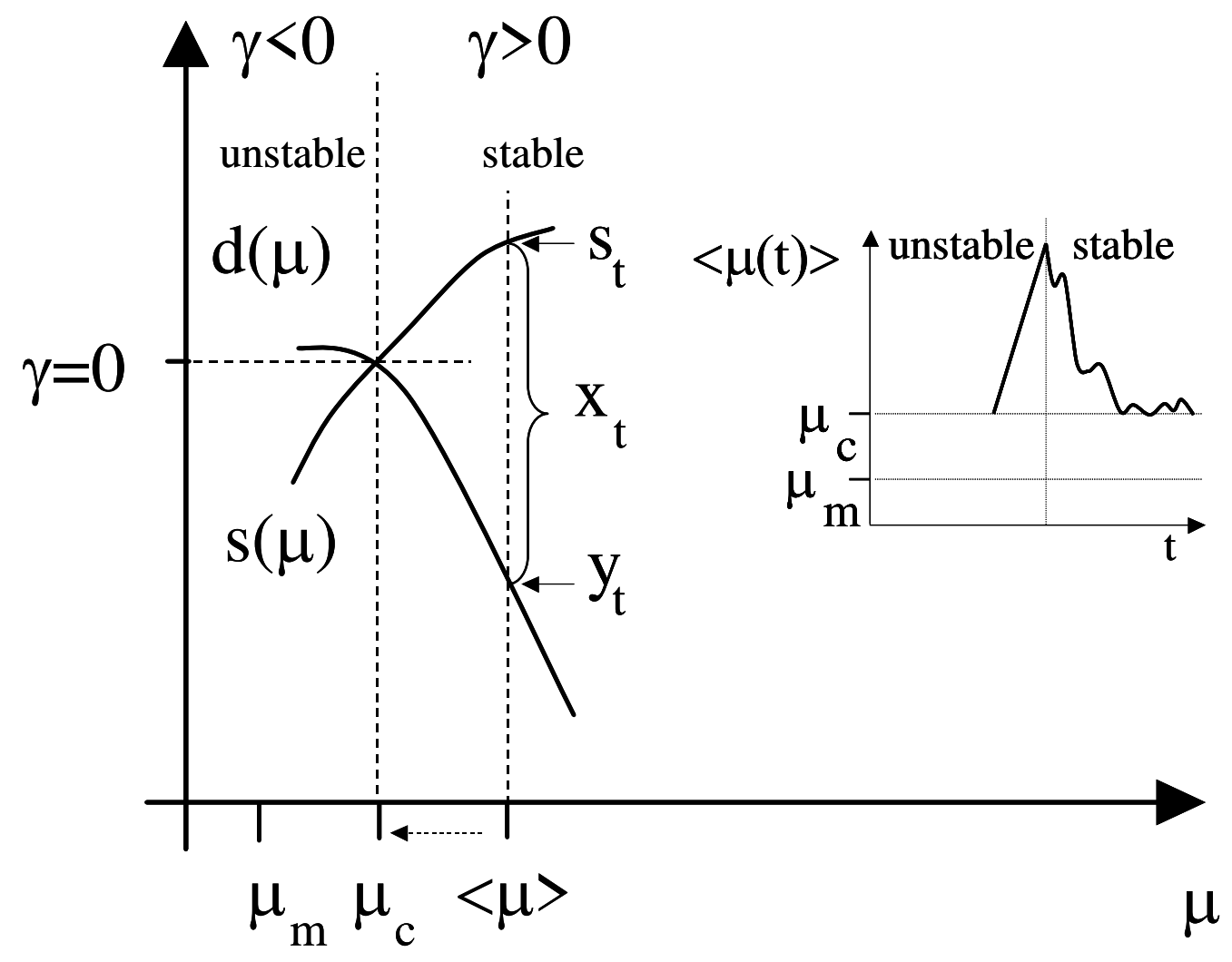

Figure 1: Displayed are the demand and supply curves as a function of the real price of a storable non-durable, where the stable and unstable regions are indicated by the dotted line at market clearing. The insert shows schematically the long term price evolution, which follows from a supply shortage event. 